MILITARY TECHNICAL COLLEGE
CAIRO - EGYPT

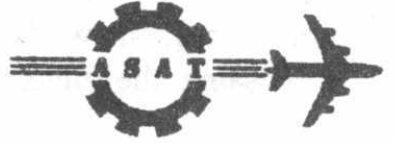

$7^{\text {th }}$ INTERNATIONAL CONF. ON AEROSPACE SCIENCES \& AVIATION TECHNOLOGY

\title{
SOLVING THE INVERSE PROBLEM OF AN AIRPLANE PLANNER MOTION VIA DIRECT APPLICATION OF HAMILTON'S LAW OF VARYING ACTION
}

\author{
A. Y. Raffie " M.B. Argoun“" and S.D. Hassan**
}

\begin{abstract}
The inverse problem for the airplane planner motion is analyzed through direct application of Hamilton's law of varying action. Using this technique one can approach the problem by solving a set of nonlinear algebraic equations only; i.e. no differential equations are needed. In this case the mass center trajectory and the required controls are approximated as a truncated power series. Direct application of Hamilton's law establishes the governing relations between those input and output coefficients. The problem is then solved in the coefficient domain to obtain the unknown control coefficients. The problem is formulated and solved for both time dependent and space dependent specified trajectories. Two illustrative numerical examples are provided to demonstrate the technique.
\end{abstract}

\section{KEY WORDS}

Flight dynamics - Flight control- Analytical dynamics, Hamilton's Law.

\section{1- INTRODUCTION}

The inverse problem means obtaining the controls required for the airplane to perform a specified maneuver. This problem is nalyzed in this paper for the case of planner motion through direct application of Hamilton's law of varying action. This novel technique allows one to obtain a solution to this problem through solving a set of nonlinear algebraic equations only i.e. no differential equations are needed.

In the last two decades, a large amount of work was done in the field of solving dynamic system problems usiing alozeb:aic equations. These methods are generally based upon assuming a certain form for the solution and employing a certain variational principle. Direct application of Hamilton's law of varying action is one of those methods. Considering the control as an inverse response problem, few works are published in the control field utilizing this direct method.

Moreover, particular interest in the inverse problem of an airplane motion was observed during the last ten years. The presnt work combines these two fields by employing direct application of Hamilton's law to the problem of airplane maneuver syntheses. In this paper, the analysis is confined to the motion in a vertical plane.

In reference [1], a spatially discrete non linear dynamic system is required to follow a specified path, and the required control is evaluated through solving a set of algebraic equations. The so called weak Hamiltonian finite element method which produces also a set of algebraic equation, was applied in an optimal control probleni in references [2] and [3]. Reference [2] demonstrates this technique for particle motion, while reference [3] confines the attention to vertical plan motion of a launch vehicle. In both cases on!y the mass point trajectory was considered. In reference [4], one of the present authors succeeded in modeling control systems using this direct technique. As an application, a technique resembling model following was introduced.

Another important field of interest is the inverse problem of aircraft notion, and specifically the rnotion in the vertical plane. Reference [5] presents a through analysis of the inverse problem for aircraft motion. This reference introduces a systematic and straight forward algorithm for this problem for both trajectory specification and attitude maneuver using the classical differential equation

\footnotetext{
- Graduat student.

"• Professor, Dept. of Aerospace Engineering, Cairo University.
} 
technique. In references [7], a general and simple formulation of the aircraft program motion is presented and applied to a square loop prescribed maneuver.

In the field of direct application of Hamilton's law for the control problem, most of the work is applied to a particle behavior or distributed parameter systems while rigid body applications are generally not considered. Although the concept may be the same, the rotational motion and the associated coordinate system relations require special consideration.

In the present work both the rectilinear and rotational motion for airplane inverse problem are considered. All the rectilinear and angular displacements as well as the associated forces and moments are represented in a polynomial form. The method accepts a path specification in both analytic and discrete points forms. The mathematical derivation is also develped for both time dependent and space deprendent specified trajectories. The displacement polynomial coefficients are obtained through curve ficting or interpolation techniques. Then through direct application of Hamilton's law of varying action a set of algebraic equation is formulated to relate the displacement coefficients to those of the resiltant forces and moments. Solution of this set of algebraic equations yields the corresponding p'seudo-control coefficients. By the pseudo-controls we mean the components of the resultant external forces and the pitching moment. They are so called because they are the direct reasons for the airplane rectilinear and rotational motion. In order to keep the equations in its algebriac form the solution is then performed in the coefficient domain. This is done through the nonlinear relations between the pseudo-controls and their physical components to obtain the main physical controls which are the final solution of the problem, namely; the thrust force $T$ and the elevator deflection $\delta \mathrm{E}$.

The paper is arranged as follows: in section 2 , the proposed theoretical approach is introduced for the case of time dependent trajectory. Section 3 is devoted to develop the technique for the case of space dependent trajectory. Two illustrative examples for both the cases are presented at the end of each section. Because of the particular characteristics of the circular loop maneuver, section 4 is devoted to treat this type of maneuver. Through two numerical examples the two ways of trajectory specification are implemented to solve the inverse problem for the circular loop, while the associated problems are demonstrated. A verification for the solutions is done in section 5 through comparison with the differential equation method. Finally, section 6 includes a discussion of the results and the concluding comments .

\section{2- THEORETICAL APPROACH}

The motion of the airplane in a vertical plane could be completely described with the help of the classical inertial $(x, y)$, body fixed $\left(x_{b}, y_{b}\right)$ and wind $\left(x_{w}, y_{w}\right)$ coordinate systems as shown in Fig. 1 . Referring to this figure the position and orientation of the airplane is completely determined by the coordinates $\mathrm{x}$ and $\mathrm{z}$ besides the pitching angle $\vartheta$.

For traditional airplane, the control variables are the magnitude of the thrust vector $|T|$ and the elevator deflection angle $\delta \mathrm{E}$. These two parameters control the aircraft in the three coordinates $\mathrm{x}, \mathrm{z}$ and $\vartheta$. In this section our interest is for the case of time dependent specified trajectory. That is, the coordinates $\mathrm{x}, \mathrm{z}$ and $\vartheta$ or some of them are specified as functions of time. In general, one can't specify all these three coordinate at the same time. Briefly, this is because as shown in Fig. 1, the angle between the velocity vector $V$ and the airplane $x$-axis is the angle of attack $\alpha$. But the velocity vector is completely specified if the coordinate rates $x^{\circ}$ and $z^{\circ}$ are given. This means specifying $x, z$ and $\theta$ as functions of time is an implicit specification of the angle of attack $\alpha$. This causes a conflict, as the aerodynamic derivatives make this angle the main factor causing the cross-coupling between the force and moment equations. This will be shown in detail next when the equations of motions are developed. Following the previous analysis, two out of the three coordinates $x, z$ and $\theta$ could be specified as functions of time and the inverse problem is to find out the two controls $\mathrm{T}$ and $\delta \mathrm{E}$ in order to realize the specified requirements. Specifying the trajectory as $x=x(t)$ and $z=z(t)$ is the most common case. In order to develop the airplane model via direct application of Hamilton's law, this law is written as:

$$
\delta \int_{t_{0}}^{t_{f}}(T+W) d t-\sum_{i=1}^{a} \frac{\partial T}{\partial \dot{q}_{i}} \delta q i||_{t_{0}}^{t_{f}}=0
$$


where $T$ is the kinetic energy, $W$ is the work done and the $q$ 's are the generalized coordinates. For the planner motion the kinetic energy is:

$$
T=\frac{1}{2} m \dot{x^{2}}+\frac{1}{2} m \dot{z}^{2}+\frac{1}{2} I \dot{\vartheta^{2}}
$$

Where $m$ is the mass of the airplane and $l$ is the moment of inertia about $y_{b}$ - axis. The only nonconservative force in this system is the gravity force $\mathrm{mg}$, and thus, it could be contained into the scalar function $\mathrm{W}$ which represents the work done as

$$
W=m g z
$$

The other forces acting upon this system are the thrust $T$, the drag $D$ and the lift $L$ forces. Besides, there is also, the aerodynamic pitching moment $M$. The inertial coordinates $x$ and $z$ and the pitch angle $\vartheta$ are chosen to be the generalized coordinates used with Hamilton's law. Thus the corresponding generalized force should be the resultant of all the forces in both $\mathrm{x}$ and $\mathrm{z}$ directions $\mathrm{X}$ and $\mathrm{Z}$ in addition to the pitching moment $\mathrm{M}$. These three generalized forces are the natural reason for the motion, and thus are called the pseudo-controls. These pseudo-controls are mainly non conservative forces and could not be introduced inside the function W. Instead, the variation of the virtual work of these forces could be added to the variation of the workdone $W$. Hence, the total variation of the work done becomes

$$
\delta \mathrm{W}_{\mathrm{t}}=\delta \mathrm{W}_{\mathrm{cf}}+\mathrm{X} \delta \mathrm{x}+\mathrm{Z} \delta \mathrm{z}+\mathrm{M} \delta \vartheta
$$

Substituting Equations (2), (3)and (4) into Equation (1), and considering the kinetic energy variation:

$$
\begin{aligned}
& \int_{\mathrm{t}_{0}}^{\mathrm{t}_{\mathrm{f}}}[\mathrm{m} \dot{\mathrm{x}} \delta \dot{\mathrm{x}}+\mathrm{m} \dot{\mathrm{z}} \delta \dot{\mathrm{z}}+\mathrm{I} \dot{\vartheta} \delta \dot{\vartheta}+\mathrm{x} \delta \mathrm{x}+(\mathrm{z}+\mathrm{mg}) \delta \mathrm{z}+\mathrm{M} \delta \vartheta] \mathrm{dt} \\
& -\left.[\mathrm{m} \dot{\mathrm{x}} \delta \mathrm{x}+\mathrm{m} \dot{\mathrm{z}} \delta \cdot \dot{\mathrm{z}}+\mathrm{I} \dot{\vartheta} \delta \dot{\vartheta}]\right|_{\mathrm{t}_{0}} ^{\mathrm{t}_{\mathrm{f}}}=0
\end{aligned}
$$

The main objective of this work is to solve the inverse problem utilizing purely algebraic equations. Substituting the ingredients of the pseudo-controls with their associated nonlinearities will complicate the integration processes needed to produce those algebraic equations. Thus, the solution is done here in two stages. The first stage is done for the pseudo-controls which keeps to Equation (5) its linear form. As will be shown later, this will produce a set of linear algebraic equations to be solved for pseudo-control coefficients. In the second stage a set of nonlinear algebraic equations is formulated to govern the relation between the pseudo-controls and their ingredients so that the physical controls $\delta \mathrm{T}$ and $\delta E$ may be obtained.

The formulation of the first stage of the solution is now explained. The trajectory is specified in the form of

$$
\mathrm{x}=\mathrm{x}(\mathrm{t}) \quad ; \quad \mathrm{z}=\mathrm{z}(\mathrm{t}) \quad ; \text { to } \leq \mathrm{t} \leq \mathrm{t}_{\mathrm{f}}
$$

the whole observation time $\left(t_{f}-t_{0}\right)$ is discretized into set of time intervals $t^{*}$ 's, and the non dimensional time $\tau$ inside each interval is introduced as

$$
\tau=\frac{t}{t^{*}}
$$

The problem is solved using the proposed technique within each time interval successively. That is, the end conditions of any interval is used as the initial conditions to the next one. Thus, all the next analysis is considered to be done within the working time interval .

The generalized coordinates and their variations are assumed to be represented by a truncated power series in the non dimentional time. e.g. for $\mathrm{x}$ we assume

$$
\begin{aligned}
& \mathrm{x}=\mathrm{A}_{x 0}+\mathrm{A}_{x 1} \tau+\sum_{i=2}^{N} \mathrm{~A}_{\mathrm{xi}} \tau^{\mathrm{i}} \\
& x^{\prime}=A_{x 1}+\sum_{i=2}^{N} i A_{x t} \tau^{i-1}
\end{aligned}
$$

The initial position and velocity are known and thus their variations vanish. Hence, 


$$
\begin{aligned}
& \delta x=\sum_{j=2}^{N} \delta A_{x j} \tau^{j} \\
& \delta x^{\prime}=\sum_{j=2}^{N} j \delta A_{x . j} \tau^{j-1}
\end{aligned}
$$

The pseudo-controls are, also assumed to be represented by a trunacted power series. Thus, for the component $\mathrm{X}$, we assume

$$
X=\sum_{k=0}^{M} B_{x k} \tau^{k}
$$

Substituting Equations (9), (10) and the similar equations of the other coordinates into Equation (5)

$$
\begin{aligned}
& \int_{0}^{1}\left\{\frac{m}{t_{*}^{2}}\left(A_{x 1}+\sum_{i=2}^{N} i A_{x i} \tau^{i-1}\right)\left(\sum_{j=2}^{N} j \tau^{j-1} \delta A_{x j}\right)+\frac{m}{t_{*}^{2}}\left(A_{z 1}+\sum_{i=2}^{N} i A_{z i} \tau^{i-1}\right)\left(\sum_{j=2}^{N} j \tau^{j-1} \delta A_{x j}\right)\right. \\
& +\frac{I}{t_{*}^{2}}\left(A_{9 i}+\sum_{i=2}^{N} i A_{9 i} \tau^{i-1}\right)\left(\sum_{j=2}^{N} j \tau^{j-1} \delta A_{9 j}\right)+\left(\sum_{k=0}^{M} B_{x k} \tau^{k}\right)\left(\sum_{j=2}^{N} \tau^{j} \delta A_{x j}\right)+ \\
& \left.\left(\sum_{k=0}^{M} B_{z k} \tau^{k}+m g\right)\left(\sum_{j=2}^{N} \tau^{j} \delta A_{z j}\right)+\left(\sum_{k=0}^{M} B_{M k} \tau^{k}\right)\left(\sum_{j=2}^{N} \tau^{j} \delta A_{9 j}\right)\right\} t_{*} d t- \\
& {\left[\frac{m}{t_{*}}\left(A_{x 1}+\sum_{i=2}^{N} i A_{x i} \tau^{i-1}\right)\left(\sum_{j=2}^{N} \tau^{j} \delta A_{x j}\right)+\frac{m}{t_{*}}\left(A_{z t}+\sum_{i=2}^{N} i A_{z i} \tau^{i-1}\right)\left(\sum_{j=2}^{N} \tau^{j} \delta A_{z j}\right)+\right.} \\
& \left.\frac{I}{t_{*}}\left(A_{91}+\sum_{i=2}^{N} i A_{9 i} \tau^{i-1}\right)\left(\sum_{j=2}^{N} \tau^{j} \delta A_{9 j}\right)\right] 1
\end{aligned}
$$

considering the arbitrariness of the variations $\delta \mathrm{A}_{\mathrm{xi}}, \delta \mathrm{A}_{\mathrm{zi}}$ and $\delta \mathrm{A}_{\vartheta \mathrm{i}},(\mathrm{i}=2,3, \ldots \mathrm{N})$, their multiplicands must equal zero, that is

$$
\delta A_{x j} \neq 0,(j=2,3 \ldots . . N)
$$

makes

$$
\begin{aligned}
& \int_{0}^{1}\left\{\frac{m}{t_{*}^{2}}\left(A_{x i}+\sum_{j=2}^{N} i A_{x l} \tau^{i-1}\right)\left(\sum_{j=2}^{N} j \tau^{j-1} \delta A_{x j}\right)+\left(\sum_{k=0}^{M} B_{x k} \tau^{k}\right)\left(\sum_{j=2}^{N} \tau^{j} \delta A_{x j}\right)\right\} t_{*} d t- \\
& {\left.\left[\frac{m}{t}\left(A_{x 1}+\sum_{i=2}^{N} i A_{x i} \tau^{i-1}\right)\left(\sum_{j=2}^{N} \tau^{j} \delta A_{x j}\right)-\right]\right|_{0} ^{1}=0}
\end{aligned}
$$

and the same for $\delta A_{z j} \neq 0, \delta A_{\Im j} \neq 0,(j=2,3 \ldots . . N)$. Carrying out the integration and with some manipulation the following linear algebraic equations are obtained.

$$
\begin{aligned}
& \sum_{i=2}^{N} \sum_{j=2}^{N} \frac{i(i-1)}{i+j-1} A_{x i}=\frac{t^{2}}{m} \sum_{k=0}^{M} \sum_{j=2}^{N} \frac{1}{k+j+1} B_{x k} \\
& \sum_{i=2}^{N} \sum_{j=2}^{N} \frac{i(i-1)}{i+j-1} A_{z i}=\frac{t^{2}}{m} \sum_{k=0}^{M} \sum_{j=2}^{N} \frac{1}{k+j+1} B_{z k}+t \cdot g \sum_{j=2}^{N} \frac{1}{j+1} \\
& \sum_{i=2}^{N} \sum_{j=2}^{N} \frac{i(i-1)}{i+j-1} A_{9 i}=\frac{t^{2}}{I} \sum_{k=0}^{M} \sum_{j=2}^{N} \frac{1}{k+j+1} B_{M k}
\end{aligned}
$$

These three equations govern the relation between the coordinate coefficients and the pseudo-control coefficients .

Specifying of the trajectory as functions of time given in Equation. (6) could be in the form of certain analytic functions or as a table of discrete values for both $\mathrm{x}$ and $\mathrm{z}$ in accordance with distinct points of time. This makes no difference, because the former case will be transformed to the later one during the treatment of the problem. Referring to Equations (12-14), whatever the way of path specification, it 
should allow to obtain the coefficients $A_{x i}$ and $A_{z i}$. This is done by an approximation of the specified functions to have polynomial forms through either a curve fitting or an interpolation technique.

It should be noticed that specifying of the path as $x=x(t), z=z(t)$ implicitly means specification of all these coordinate time derivatives. This means that specifying the path in such a way not only specify the trajectory shape but also its kinematics characteristics, namely the velocity magnitude $V$ and the flight path angle $\gamma$. Referring to Fig (1), this is clear from the following kinematics relations .

$$
\begin{aligned}
& V=\sqrt{\dot{x}^{2}}+\dot{\ddot{z}}^{2} \\
& \gamma=\tan ^{-1}\left(-\frac{\dot{z}}{\dot{x}}\right)
\end{aligned}
$$

Referring to Equations (15) and ( 16 ) any kind of curve fitting for the case of introducing the trajectory as distinct points should insure with a sufficient approximation the continuity and the smoothness of the first order derivatives of each of the coordinates, other wise the accuracy of the system is adversely affected. As a conclusion, the coefficients $A_{x i}$ and $A_{z i}(i=2, \ldots \ldots, N)$ are now available. Hence Equations 12 and 13 could be easily solved for the pseudo-control coefficients $B_{x k}$ and $B_{z k}(k=0 \ldots \ldots . M)$. This leads to the necessity of finding out the ingredients of these pseudo controls. For this planner motion the main forces acting upon the airplane is the thrust $T$, the lift $L$ and the drag $D$ as shown in Fig 1. Referring to Fig. (1) the pseudo-controls while represented in a polynomial form equal

$$
\begin{aligned}
& \sum_{k=0}^{M} B_{x k} \tau^{k}=T \cos \vartheta-D \cos \gamma-L \sin \gamma \\
& \sum_{k=0}^{M} B_{z k}=T \sin \vartheta-D \sin \gamma-L \cos \gamma
\end{aligned}
$$

As we can see, only one of the physical controls $\mathrm{T}$ is seen in Equations.(17) and (18). As it is well known the second control element $\delta \mathrm{E}$ must be found in the a aerodynamic moment $\mathrm{M}$ and may be also found in the drag and left forces. Thus, the aerodynamic forces and moment formulas should be, now, analyzed. In general, these force and moment have the following forms

$$
\begin{aligned}
& D=D(V, z, \alpha, u, \delta E) \\
& L=L(V, z, \alpha, \dot{\alpha}, q, u, \delta E) \\
& M=M(V, z, \alpha, \dot{\alpha}, q, u, \delta E)
\end{aligned}
$$

These three equations are in general nonlinear. While the pseudo-controls coefficients $B_{x k}$ and $B_{z k}$ are available, the third set of coefficients $B_{M k}$ has no. been evaluated yet. These coefficient could be obtained by solving Equation.(14) if the coefficients of pitch angle $\vartheta$ were given, in other word if $\vartheta$ were given as a function of time. But, this is not the case and what we have instead is the flight path angle $\gamma$. But referring to Fig. (1) we have

$$
\vartheta=\gamma+\alpha
$$

If $\alpha$ were given as a function of time, the coefficients of $A \vartheta_{i}(i-2 . \ldots \ldots . N)$ could be also, obtained through curve fitting or interpolation technique. Then Equation. (14) cuald be solved for the moment coefficients $B_{M k}(k=0, \ldots M)$. And thus, similar to Equations. (17) ana i 18$)$ an equation relating the pitching moment in the polynomial form to its physical representation could u nbtained. Although $\alpha$ have not been known. this equation could be written as

$$
\sum_{k=0}^{M} B_{M k} \tau^{k}=M(V, z, \alpha, \dot{\alpha}, q, u, \delta E)
$$

Now , Equations. (17), (18) and (23) are inspected to be solved for the controls $T$ and $\delta E$. The supporting equations (15), (16) and $(19 \div 22)$ should be also considered. Equation (22) is then differentiated to be 


$$
\dot{\vartheta}=\dot{y}+\dot{\alpha}
$$

where for the planner motion $q=\vartheta^{\circ}$

The final variable needs to be identified is the forward velocity along $\mathrm{x}_{\mathrm{b}}$-axis $\mathrm{u}$, regarding to Fig

(1) it's found to be

$$
\mathrm{u}=\mathrm{v} \cos \alpha
$$

Accordingly, the state of the variables are, now as follows

- The pseudo - controls coefficients $B_{X K}$ and $B_{Z K K}(k=0$,

M) has known values

-The variables $\mathrm{z}(\mathrm{t}), \mathrm{v}(\mathrm{t}), \gamma(\mathrm{t})$ and $\gamma^{\bullet}(\mathrm{t})$ are known functions of time.

- Beside the two controls $T$ and $\delta E$, the angle of attack $\alpha$ and its rate are still unknowns.

- The coefficients $B_{M k}(k=0, \ldots \ldots . . M)$ require the third Hamilton's law equation Equation. (14) to be solved, to have known vaiues.

It coul $i$ be easily seen that the angle of attack $\alpha$ is the key variable of these equations. that is knowing this 'rariable as a function of time reduces the number of unknowns to be only the physical coritrols $T$ and $\delta E$. In other words, Equations (17), (18) and (23) could be solved for $T, \delta E$ and $\alpha$. On the other hand the appearance of $\alpha$ and $q$ in these equations returns the problem back to the differential equation field which is violating our philosophy to solve the problem without any reference to differential equation theory.

To maintain this attitude, the problem is going to be continued in the so called coefficients domain. that is the equation are to be solved while all the involved variables are assumed to be introduced as a polynomial functions of the non-dimensional time $\tau$. Accordingly, and taking $\alpha$ as an example we assume

$$
\alpha=\sum_{k=0}^{M} B_{\alpha k} \tau^{k}
$$

this makes $\alpha^{\bullet}$

$$
\alpha=\frac{1}{t_{*}}\left[\sum_{k=0}^{M} k B_{\alpha k} \tau^{k-i}\right]
$$

and the same is also assumed for $T$ and $\delta E$

This representation allows to formulate a Newtonian algorithm to solve the three nonlinear equations (17), (18) and (23) while the supplementary Equation (14) is solved within this algorithm for the coefficient $B_{M k}(k=0, \ldots \ldots M)$. This could be done by assuming the angle of attack $\alpha$ as the third control, and solve the above mentioned three equations for the coefficients $\mathrm{B}_{\mathrm{Tk}}, \mathrm{BEk}_{\mathrm{E}}$ and $\mathrm{B}_{\alpha \mathrm{k}}$ $(k=0, \ldots M)$. This allows $B_{\alpha k}(k=0, \ldots \ldots . M)$ to have guessing values. Hence, through Eq. (22) the coefficients $A_{\theta i}(i=2, \ldots \ldots . n)$ could be obtained and the linear algebraic equation (14) could be solved for pitching moment coefficients $B_{M k}(k=0, \ldots \ldots m)$. this allows the right hand side of each of Eqs. (17) , (18) and (23) to have a numerical value at any instant within time and for certain guessing value of $\alpha$.

At the same time, through the known coefficients, the functions of time for the known variables and the guessed coefficients the left hand sided of each of these equation could also yield numerical values at any instant of time. The Newtonion algorithm is, thus established to find out the guessing coefficients which makes the error between the corresponding left and right hand side of each of the previously mentioned equations within the required accuracy. But we have three equations for three variables introduced in the form of $(\mathrm{M}+1)$ coefficients per each variable, this makes the unknowns to be $3 x(M+1)$.

To relate the number of equations to the number of unknowns two proposed approaches are, here in, introduced. First , as a matter of fact, each of Eqs. (17), (18) and (23) is not a single equation when the numerical values are considered. Each equation yields certain numerical value at each instant of time. Regarding this fact, a well distributed $(M+1)$ instant of time is chosen to solve $3 x(M+1)$ equations for the $3 x(M+1)$ unknowns coefficients. 
In the seccind approach, the same way of generating the pseudo-control coefficients is followed for the right har.sd side terms of the equations to be solved. This match the fact that the origin of generating the coeffic,ients in the proposed method is an approximation through either curve fitting or interpolation techritique. This means that, a sufficient number of time instants are chosen to evaluate numerical values fo's each of the right hand side of the equations. Then through curve fitting a vector of coefficients with $(M+1)$ order is constructed to each equation. Consequently, the algorithm is established to compare these vectors of coefficients with those vectors of coefficients of the pseudo-controls already evaluated in the left hand sides of the equations

Numerical example

The proposed technique is, now applied for a hypothetical model of a small jet trainer airplane. The parameters of this model is introduced in Appendix ( C ) in Reference [9]. This hypothetical model is asked to perform a maneuver given as

$$
\begin{aligned}
& x(t)=\frac{v_{0}}{\omega_{x}} \sin \omega_{x} t \\
& z(t)=-4 * \sqrt{t}\left(\cos \frac{3 \pi}{T_{b}} t-9 \cos \frac{\pi}{T_{b}}+8\right)
\end{aligned}
$$

where the parameters of these equations are chosen so that the airplane may enter the maneuver from a steady state straight line flight with the nominal velocity $V_{0}$. The observation time was taken to be 60 seconds. This period was discretized into 30 intervals and the displacements $\mathrm{x}$ and $\mathrm{z}$ are approximated with a fifth order polynomial. This makes the pseudo controls approximation to be of a third order one.

Figs. (17) and (18) show the specified trajectory given as $x(t)$ and $z(t)$, while the spatial shape in the $x-z$ plane is shown in Fig. 2. This shape is chosen to simulate an avoidance of two successive obstacles .

The utilized model assumes the thrust force to be evaluated through a thrust coefficient $C_{T}$. Thus, controlling of the thrust is also assumed to be through varying of this thrust coefficients. This yieids

$$
T=\frac{1}{2} \rho v(t)^{2} S_{r}\left[C_{T}+\Delta C_{T}(t)\right]
$$

The time history for the angle of attack, the variation in the thrust coefficient $\Delta C_{T}$, and the elevator deflection $\delta E$ are depicted in Figs. (3) (4) and (5) respectively. The nominal value of the thrust, the variation in the magnitude of the thrust, and the total thrust are shown together in fig (6). As an auxiliary output, the behavior of the pitch angle $\theta$ is shown in Fig. (19)

\section{3- SPACE DEPENDENT SPECIFIED TRAJECTORY}

The space dependent specified trajectory means to introduce the required path in the form of a relation between the space coordinates, in our case $x$ and $z$. That is the airplane is asked to follow certain spatially determined trajectory regardless of time considerations. Thus, the spatial path is specified as the function .

$$
F(x, z)=0
$$

The technique applied in this case is similar to direct simulation of the airplane motion, while the differential equations are replaced by our algebraic equation model. The main idea in this case is to carry out the simulation to the airplane motion while the path specification equation is considered as a constraint imposed on this motion .

The main equations employed for simulation are the three equations (12), (13) and (14), which are complemented by Eqs $(17),(18)$ and (23), respectively. Eq. 35, is the constraint equation. Beside the constraint, the kinematics relations given by Eqs (15), (16) and (22) introduce the system states as functions of just three unknowns. It could be seen that the three pairs of equations simulating the airplane motion are representing the problem in the time domain. On the other hand, the constraint is considering the space representation of the problem.

As there is no correspondence between the time and the space till now, the coefficients $A_{x i}$ and $A_{z i}$ ( $i$ $=2, \ldots n$ ) of the non-dimensional time polynomials are not free and thus could be introduced as two sets of unknowns. The equations of the constraint govern the relation between these two sets at any 
instant, and don't specify any of them. This means that during the solution one can solve for one only of these two coefficients while the other could be found through the constraint equation. In the same sense, the kinematics relation (15) allows the velocity $v(t)$ to be found if $x(t)$ and $z(t)$ are given. This adds just one unknown to the three previously mentioned ones $\alpha, T$ and $\delta \mathrm{E}$. Thus, it is required to specify one of the control variables as a function of the system states.

The physical consideration of the problem nominates the thrust to be that variable to allow the maneuver in the vertical plane through elevator deflection $\delta \mathrm{E}$. That is the thrust is to be specified as a function of any of the system states or could be assumed constant value. Hence in the same manner as the time dependent specified trajectory the three main equations are solved for either $\mathrm{x}$ or $\mathrm{z}, \alpha$ and $\delta$ $E$. This choice of the variable makes the problem seems to be as a mixture of a direct and an inverse one. However the target is to follow up a specified path and thus, it is a typical inverse response problem .

Inspecting. Hamilton's law equations, it is shown that they are considering the coefficients starting from the quadratic term and the higher order ones. This requires the coefficients of the zero order and the first order terms to be given a priori. As a mater of fact these coefficients are the initial conditions of the problem and they must be given for the first interval to continue with the solution.

The last point to be clarified is how does the time and the space correlate during the solution. This is done by choosing of certain period of time to be the first interval regardless any space consideration. The algorithm is , then employed to solve this first interval for the coefficients of the variables $(x, z$ or $\mathrm{v}), \alpha$ and $\delta \mathrm{E}$. The results concerning either of $\mathrm{x}$ or $\mathrm{z}$ could be used to find out the corresponding final values of those variables. Then, as usual, the end conditions of each interval are considered as the iritial conditions for the next one. Then a time period value is chosen to the next interval and the solution is completed as the previous one. The stopping condition is reached if the end value of the forward rang $x$ is greater than that of the specified trajectory

\section{Numerical example}

This illustrative example is applied for airplane of reference [9]. The specified path is assumed to have the form: $z=A e^{\lambda_{x}} \cos \omega_{x}$, where $A=15 \quad \mathrm{~m} ; \quad \lambda_{\mathrm{x}}=2 \times 10^{-4} ; \quad \omega_{\mathrm{x}}=1.3 \times 10^{-3}$

The trajectory is observed up to 12 kilometers, and the shape of this trajectory is shown in Fig.(T)

Equal time intervals are used for this trajectory, where $t_{*}$ is chosen to be 3 secs. As the thrust force in this model is evaluated through the thrust coefficient $C_{T}$, this value is assumed to be constant. then the thrust becomes

$$
T=1 / 2 \rho v(t)^{2} S_{r} C_{T}
$$

The time of the maneuver was found to be 51 secs. for 17 intervals. The behavior with respect to $x$ coordinate for the angle of attack $\alpha$, the velocity $\mathrm{V}$ and the elevator angle $\delta \mathrm{E}$ are depicted in figs. ( 8), (9) and 10 , respectively. The thrust force is shown in fig. 11, where the direct proportionality to the square of the velocity is demonstrated

\section{4 - APPLICATION FOR A VERTICAL CIRCULAR LOOP:}

The flight in a vertical circular loop is one of the most common aerobatics maneuvers. Although this kind of maneuver is more or less like one of the previously mentioned cases, it will be studied, here in, specially because of its particular characteristics. The first special feature could be predicted from Eq (20), as performing a circular loop requires the velocity component $x^{\bullet}$ to have zero value twice during one cycle. This results in a singularity detection during computation. secondly, this loop could not be realized without a necessity of inverted flight. this requires special care while finding out angles during the solution. As shown this type of maneuver is considered to be the extreme of complexity for the planner motion. And, thus, it will be a good exercise to compare the performance of the two methods of trajectory specification. Hence, the circular loop maneuver is going to be analyzed through both the methods and the results are to be compared

\section{a - Frormulation of the Problem as a Time Dependent Trajectory}

Fig (12) exhibits the motion of the airplane in a circular loop where the inertial axes origin is chosen to be the center of the circle. The time dependent representation requires the coordinates 
$x$ and $z$ to be given as function of time $t$. For these parameteric equations to represent a circle they must have the form

$$
x=R \sin \omega(t) t, \quad Z=R \cos \omega(t) t
$$

where $R$ is the radius of the circle and the choicey of the function $\omega(t)$ specifies the course of velocity on the circle. As the velocity vector is always the tangent of the circle, then the centraf angle equals the flight path angle. This makes the arc- length relation to be

$$
s=\int_{t_{0}}^{t_{t}} R \omega(t) d t
$$

Now , Eqs.(28) could be introduced to the algorithm of solving the time dependent specified trajectory while the following should be considered .

- A choice of the center of the circle as the origin of the coordinate system allows detection of the quarter of flight through the sign of each of the coordinates. This identifies the inverted flight and the correction for the angle $\gamma$ could be done. As a consequence the angle $\theta$ is also corrected through the Eq ( 22 ).

- Eq.(29) insures the correspondence between time and space. hence the discretiztion of the time could be done so that the singularity points may be isolated

\section{Numerical example}

As a numerical application the air plane of reference [9], is asked to perform this maneuver for a circle of radius $R=2000 \mathrm{~m}$. The nominal velocity of $V=186 \mathrm{~m} / \mathrm{sec}$, is assumed to be kept constant all over the path. The period of the cycle is thus $T p=67.56 \mathrm{sec}$. The circle is divided into 30 sections. This make the time interval to be $t_{*}=2.252$ sec. and the angular section to be $\Delta$

$\gamma=12^{\circ}$

Solving the problem as a time dependent path produces the thrust and the elevator deflection... $\delta \mathrm{E}$ as shown in Figs ( 13 ) and ( 14 ). Besides, the angle of attack $\alpha$ is depicted in Fig (15). The behavior of the variables is considered with respect to the Flight path angle $\gamma$ to allow the comparison with the next solution of space dependent trajectory. Although we have constant velocity, Fig (16) shows this behavior as the next result will be depicted on this figure also.

\section{b. Formulation of the problem as a space dependent trajectory.}

In this case the value of the time interval is chosen and through the solution the corresponding space values are evaluated. This makes the isolation of the singularity not easy to be done. Beside that, the Cartesian representation of the problem causes very stiff behavior for the $z$-coordinate near $\gamma=0$ and $\pi$ while the $x$ - coordinate is suffering from this problem for $\gamma=\pi / 2$ and $3 \pi / 2$ where the singularity is found. This degrades, seriously the accuracy of curve fitting approximation. But referring to our algorithm we can find that the approximation is done for one of $\mathrm{x}, \mathrm{z}$ or $\mathrm{v}$ while the other two variables are evaluated through the supporting equations. Hence during the solution the flight path angle $\gamma$ is detected and the problem is alternatively solved for either $\mathbf{x}$ or $\mathrm{z}$. This means That near the singular point the problem is solved for $z$ and no singularity occurs.

The same numerical example is solved here using the constrained motion method. This approach requires the initial velocity to be given. For our model the minimum velocity allowing to perform the $2 \mathrm{Km}$ radius circle is found to be $265 \mathrm{~m} / \mathrm{sec}$, thus the velocity is chosen to be $V_{0}$ $=270 \mathrm{~m} / \mathrm{sec}$

The trajectory entrance is assumed at $x=0$ and $Z=-R$ with the velocity, of course, tangent to the trajectory. Hence $x_{0}{ }^{\circ}=V_{0}$ and $z_{0}{ }^{\circ}=0$. A time interval of $t_{*}=2 \sec$ is chosen and repeted untii the recurn to the trajectory entrance which occurs at $t=70.3 \mathrm{sec}$. The result for the angle of attack, the velocity and the elevator angle are shown in Figs (15), (16) and (14) respectively the total thrust is depicted in Fig (13).

Now, the results obtained from each case is going to be compared. Referring to Figs (15) and (14) it can be seen that the behavior of the angle of attack and the elevator deflection for the first case is milder than that of the second case. on the other hand the thrust force changes more mildly for the 
second case as show in Fig (13). This matches the sense that the problem is essentially a trajectory following one and the main task is to keep the mass center on the specified path.

As for the first case when the magnitude of the thrust is allowed to be changed it plays the role of the main executor of the maneuver through its intensive variation while the mild behavior of both the angle of attack $\alpha$ and the elevator deflection $\delta E$ is just to give the airplane the required angular position to allow the thrust components to perform the maneuver. In the second case, the advantage of variation of the magnitude of the thrust force is no longer allowed. This makes the angle of attack $\alpha$ and the elevator deflection $\delta E$ to behave more efficiently in order to employ the aerodynamic forces in the process of trajectory synthesis. However, utilizing the advantage of the thrust variation is not open as the large magnitude of the thrust and specially that negative one may not be practically realized

\section{5- VERIFIC,ATION OF THE METHODOLOGY}

To verify this way of solution, the physical controls computed through the proposed technique are plugged as an inputs to the classical differential equation model of the airplane simulation. The set of states describing the trajectory in the airplane response are then inspected. In order that our method to be verified these states should be coincide with those of the specified trajectory. However, here in, all the problem states are compared with their equivalents in the proposed method to simulate the identity of the problem

The differential equations are then integrated using fourth order Runge-kutta algorithm for each casie of the illustrative examples. All the results demonstrates an exact coincidence between the specified path and the response obtained from the diffrential equation model

The results for the numerical example of section two are introduced here. The main three states chractrizing the trajector $\mathrm{x}, \mathrm{z}$ and $\theta$ are plotted together with the corresponding results of the differential equation model. These three pairs of time histories are depicted in Figs (17-19) where the crossed line is for the differential equation solution. It could be seen that ail the results ariz almost indistinguishable.

\section{6 - CONILUDING COMMENTS}

Through direct application of Hamilton's law of varying action, and continuing with the solution in the coefficient domain, the inverse problem for airplane planner motion was solved using algebraic equations only. The method was demonstrated for both time dependent and spar,e dependent specified trajectories. An application for the complicated circular loop maneuver was also done and all the results shows that this methods is Competitive to numerical integration of the differential equation model

The results was obtained through discretization of the problem into time intervals ranges from 2 to $3 \mathrm{sec}$. The polynomial used for approximating the control variables was of fourth or fifth order. It was found that while solving the probiem as a time dependent one, that is when the time is important for the maneuver, special care should be done while designing the trajectory to avoid impractical controls specially for the thrust force. Also, while solving the problem as a space dependent one, the thrust force should be included into the solution in some manner to utilize the advantage of thrust control and to avoid unrequited large angle of attack or unfeasible elevator deflection .

This work could be extended to the airplane general motion where Hamilton's law should be developed to accept relative motion between reference frames. Also the algebraic equation model could be utilized to formulate much simpler algorithms for optimization and or imposing a constraints to obtain more feasible controls

References.

[1] $\mathrm{Oz}, \mathrm{H}$.: and Roffie, A .," Inverse Response problem (control) of Dynamic systems Via Hamilton's law ", comput Methods Appl. Meth . Engrg . 62, (1987) pp (17-26)

[2] Hodges, D. H. , and Bless, R. R., "Weak Hamiltonian Finite Element Method For Optimal Control Problems", Journal of Guidance, Control, and Dynamics, Vol 14, No 1, (1991), pp (148-156).

[3] Hodges, D.H, Bless, R. R., Catise, A. J., Leung, M., "Finite Element for Optimal Guidance of an advanced Launch Vehicle, Journal of Guidance, Control, and Dynamics, vol 15, No 3, (1992), pp (664-671). 
[4] Raffie , A .Y. ," A modeling Via Hamilton's law with application to a model following resembling technique," comut. Methods Appl. Mech. Engrg. 123, (1995) pp (63-79)

[5] kato.$\theta$. and sugiural I." An Interpretation of Airplane General Motion and control as Inverse problem ", journal of Guidance, control, Dynamics, vol g. March - April ( 1986 ) pp ( 198 - 104 )

[6] Blajer, W., " Modeling of aircraft program motion with application to circular loop simulation ", Aeronaut , J, Aug. . Sep . ( 1988 ). 92, pp (289-296)

[7] Blajer,W. and parczewski,J., "Aircraft program Motion Along a predetermined Trajectory Part I Mathematical modelling," Aeronaut. J. Feb. (1990), 94, pp (53-58).

[8] Etkin, B, "Dynamics of atmospheric Flight, Wiley. New York, 1972

[9] Roskam , J ., " Airplane Flight Dynqmics and Automatic Flight Controls, Roskam Aviation and Eingineering Corporation, " , USA , ( 1979 )

[10] Miero vitch,L.," Methods of analytical Dynamics", 1st Ed., Mc Graw-Hill, New York, (1970)

[11] Hamilton, W.R., "On a General Method in Dynamics",. Trans .R. soc. Lond . 124, (1834), pp (247-308)

[12] Hamilton, W.R., Second Essay on a general Method in Dynamics, Trans. R. soc. Lond. 125, (1835), pp (95-144). 


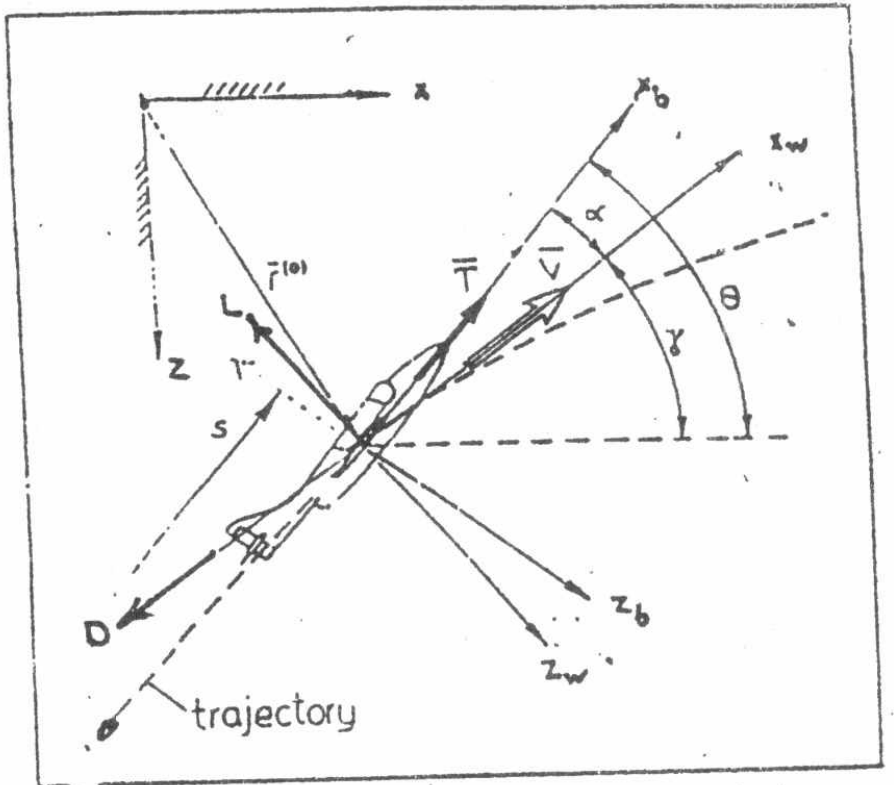

Figure 1. Aircraft trajectory flight (plane motion).

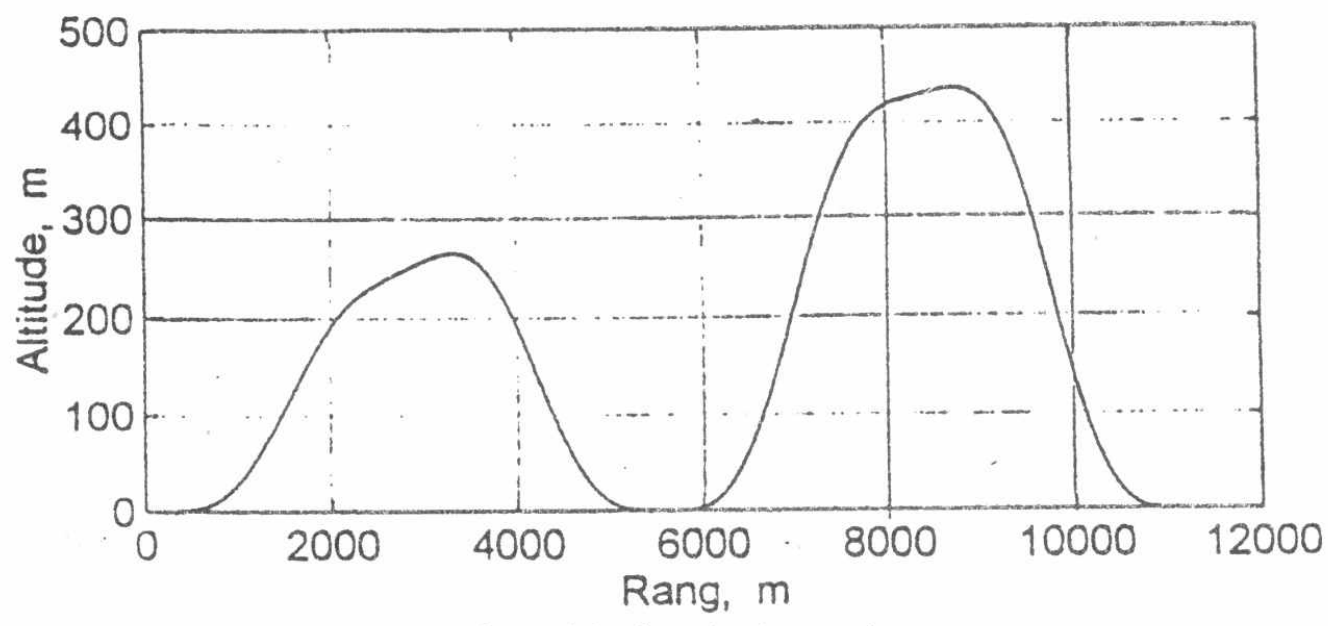

Fig.2. Specified trajectory shape.

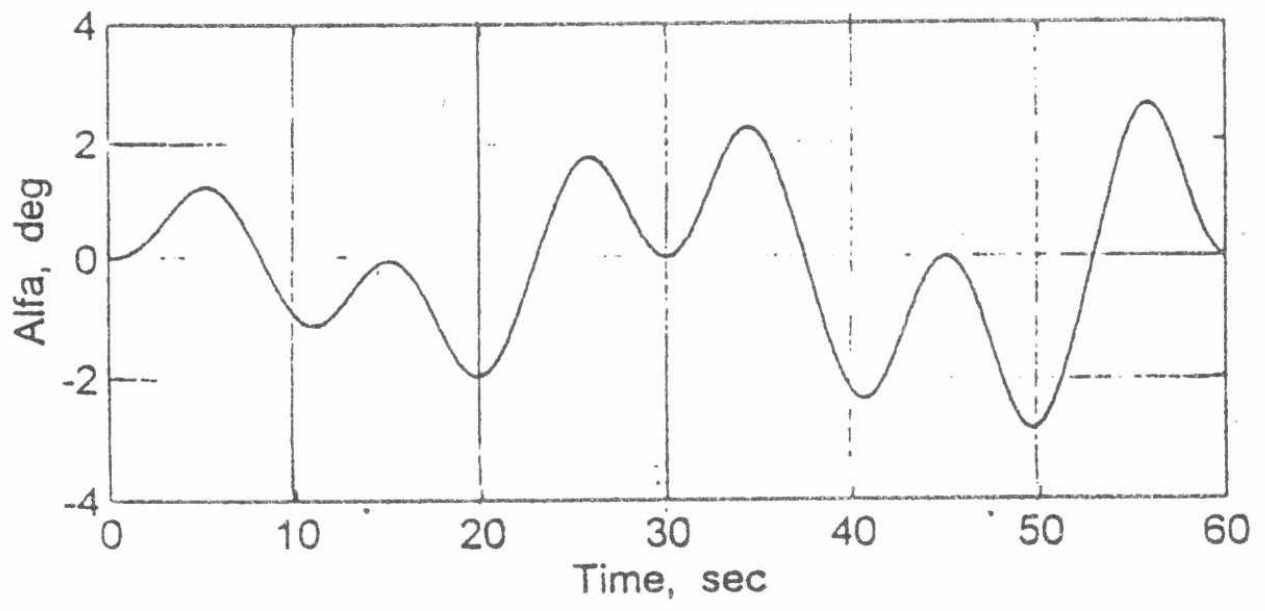

Fig.3. Angle of attack time history. 


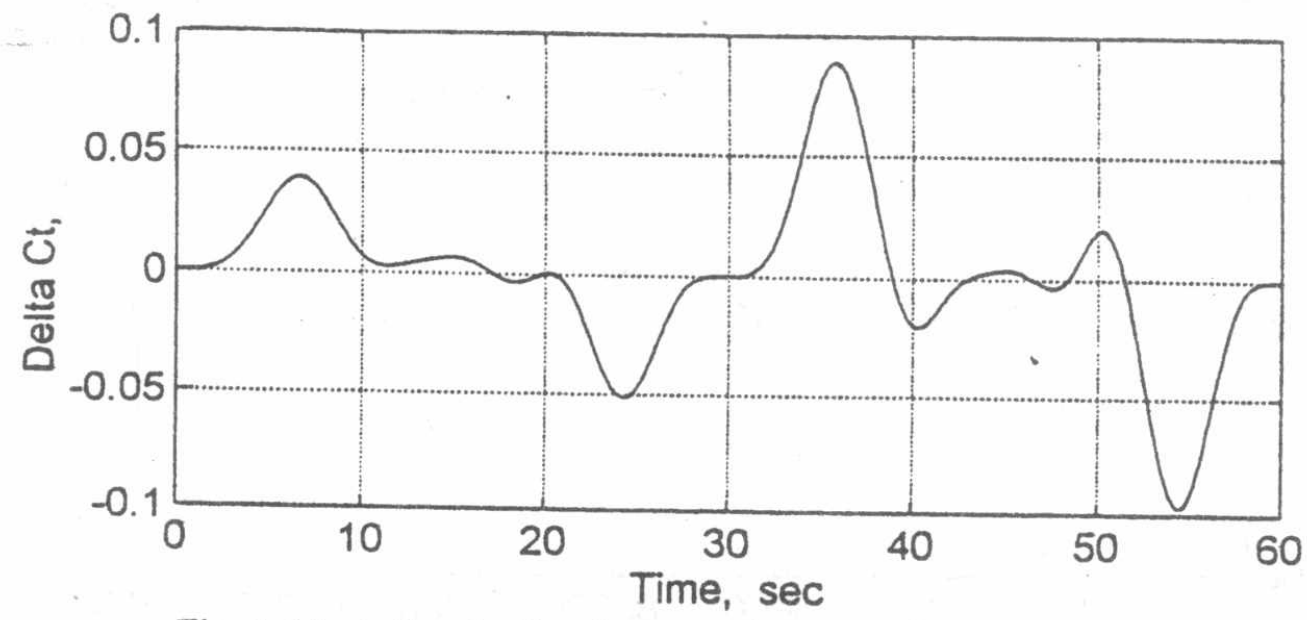

Fig.4. Variation in the thrust coefficient vs time.

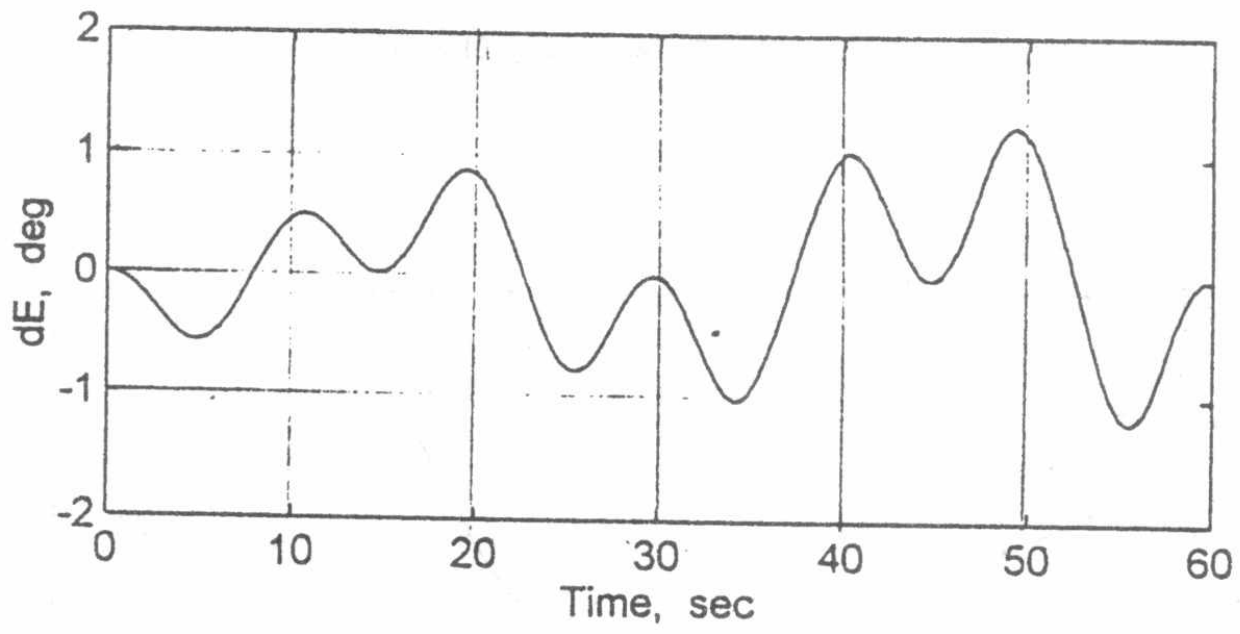

Fig.5. Elevator deflection time history.

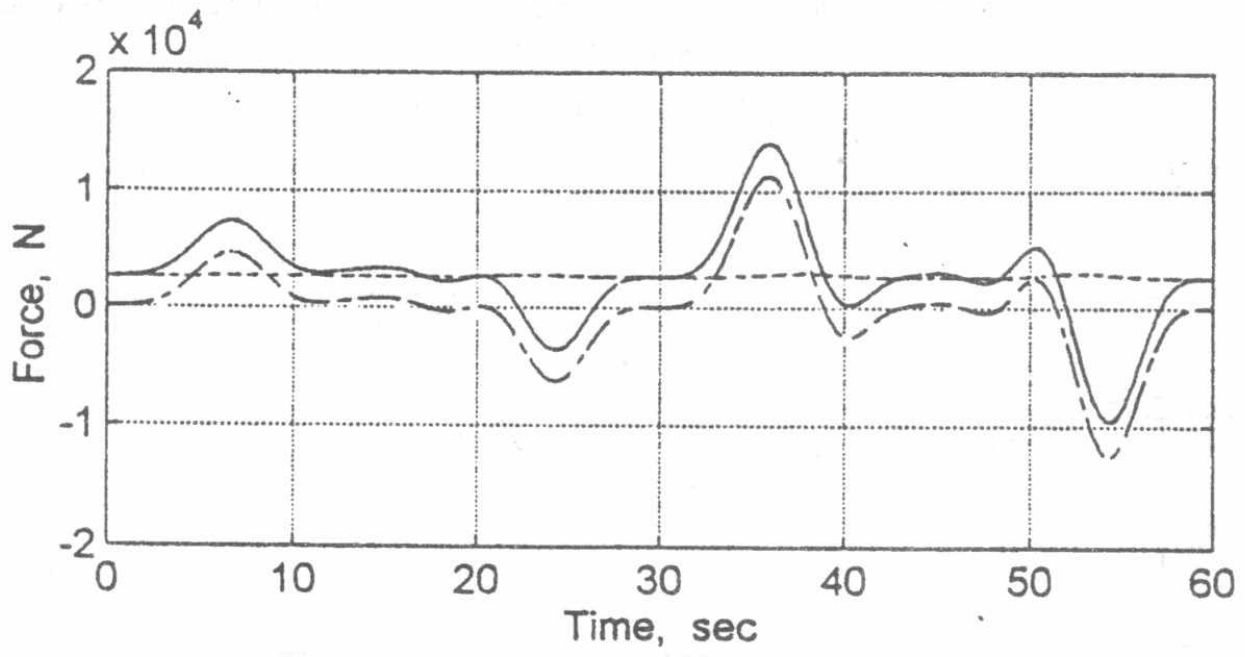

Fig.6. Total thr, nominal thr and variation in thrust vs time. 


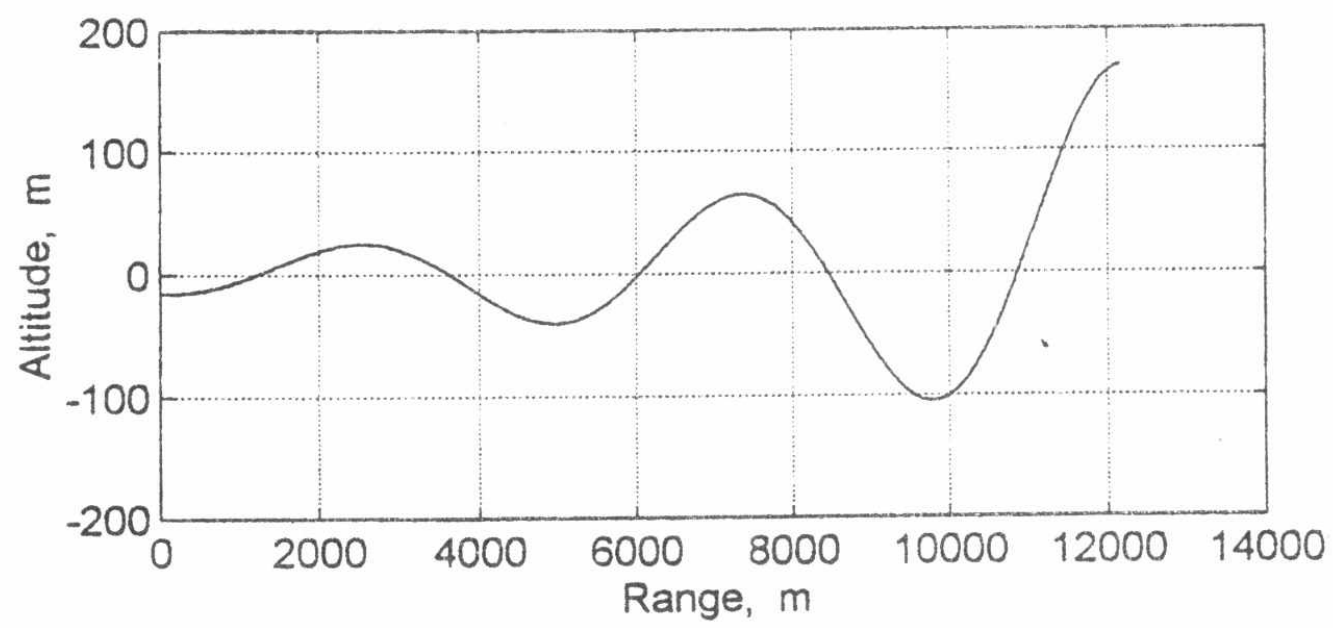

Fig.7. Specified trajectory shape.

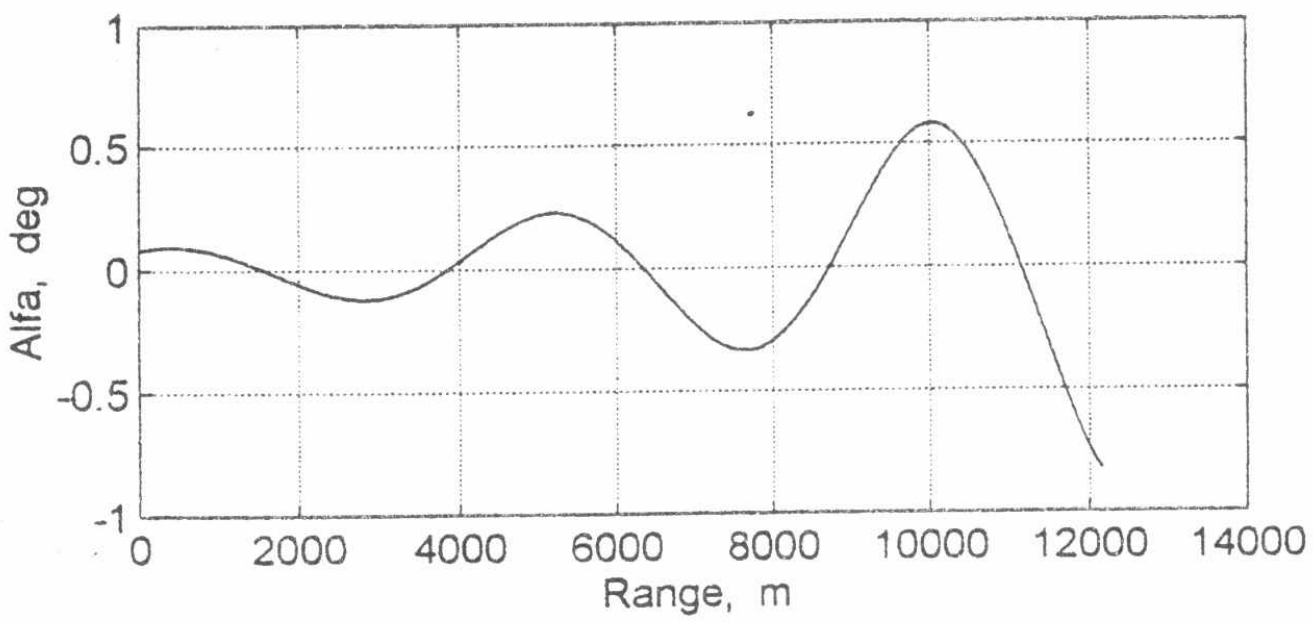

Fig. 8. Angle of attack vs range.

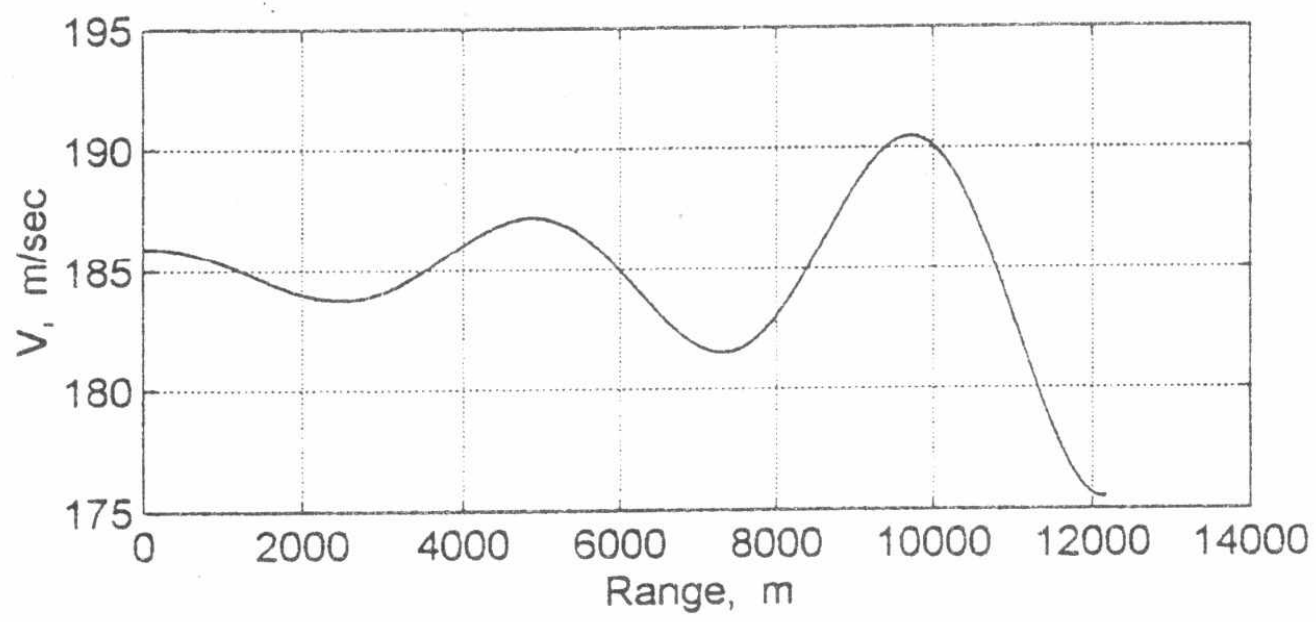

Fig.9. Velocity vs range. 
Proceedings of the $7^{\text {th }}$ ASAT Conf. 13-15 May 1997

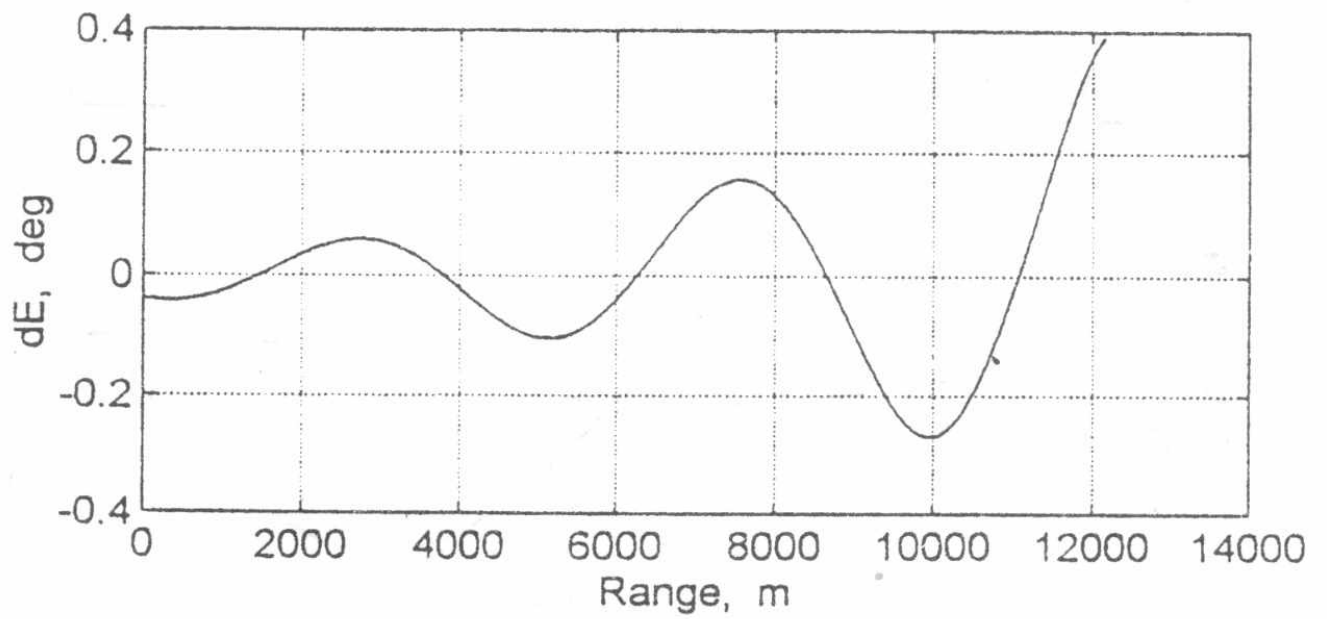

Fig.10. Elevator deflection vs range.

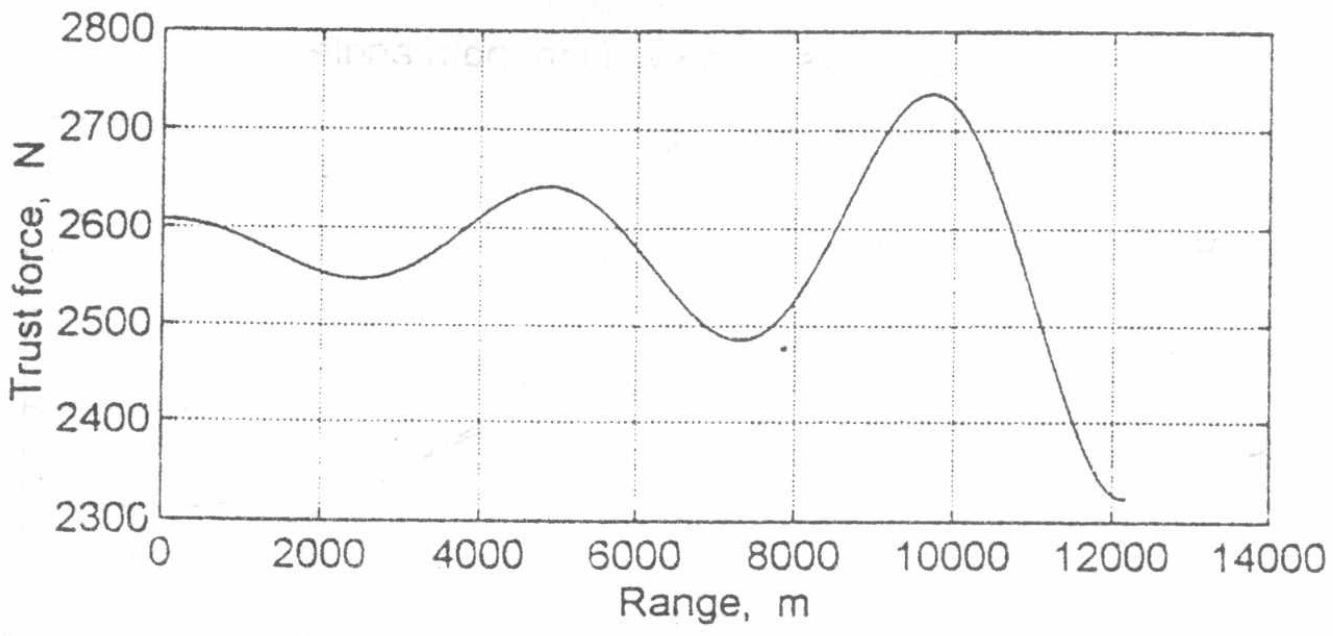

Fig.11. Thrust force vs range.

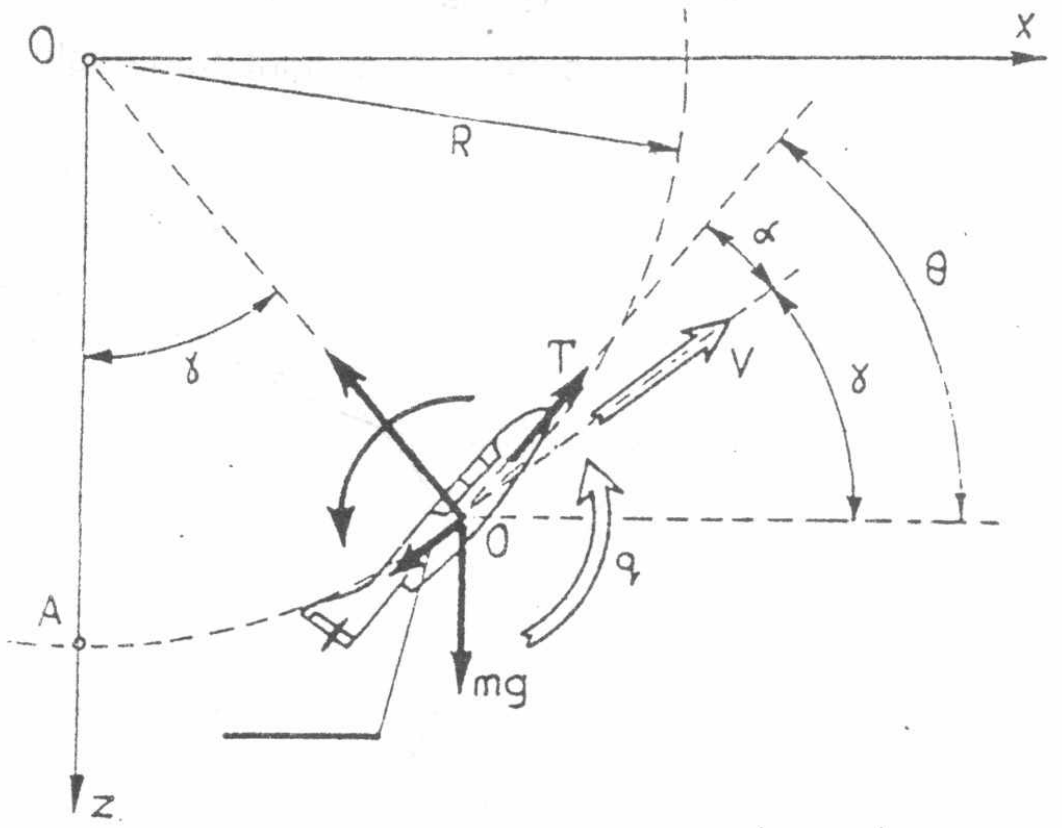

L Fig.12. Airplane motion in circular loop 
Proceedings of the $7^{\text {th }}$ ASAT Conf. 13-15 May 1997

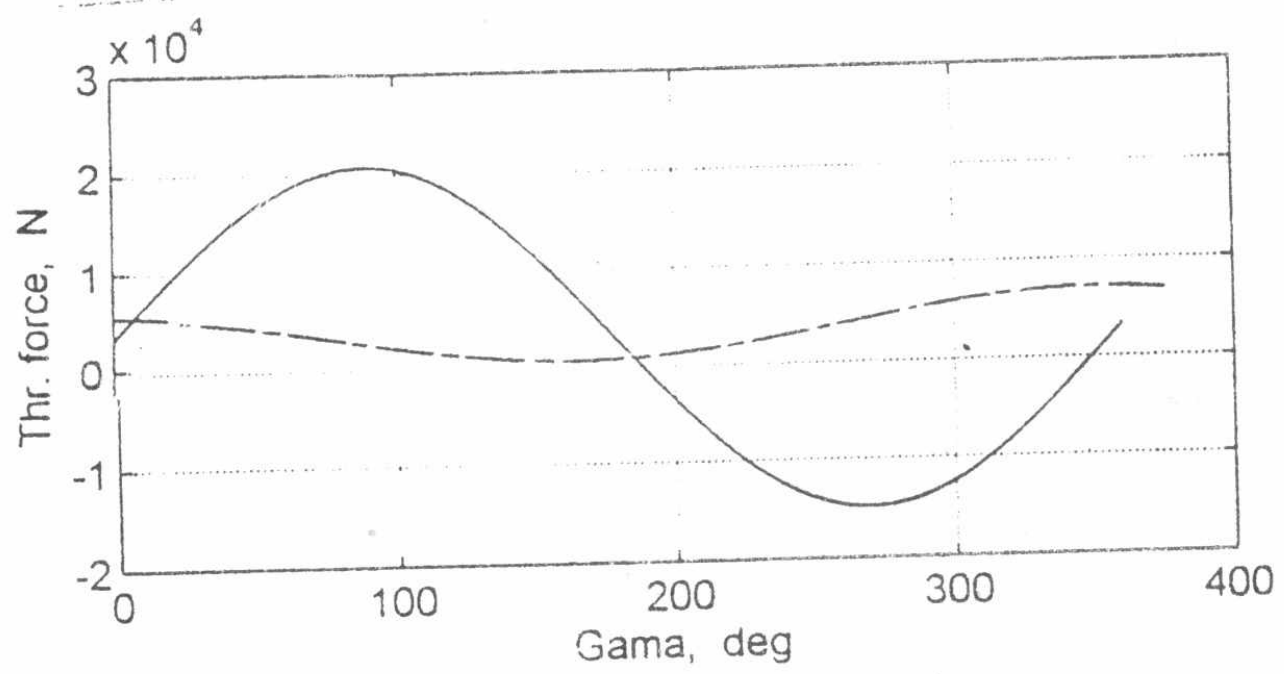

Fig. 13. Thrust force vs flight path angle.

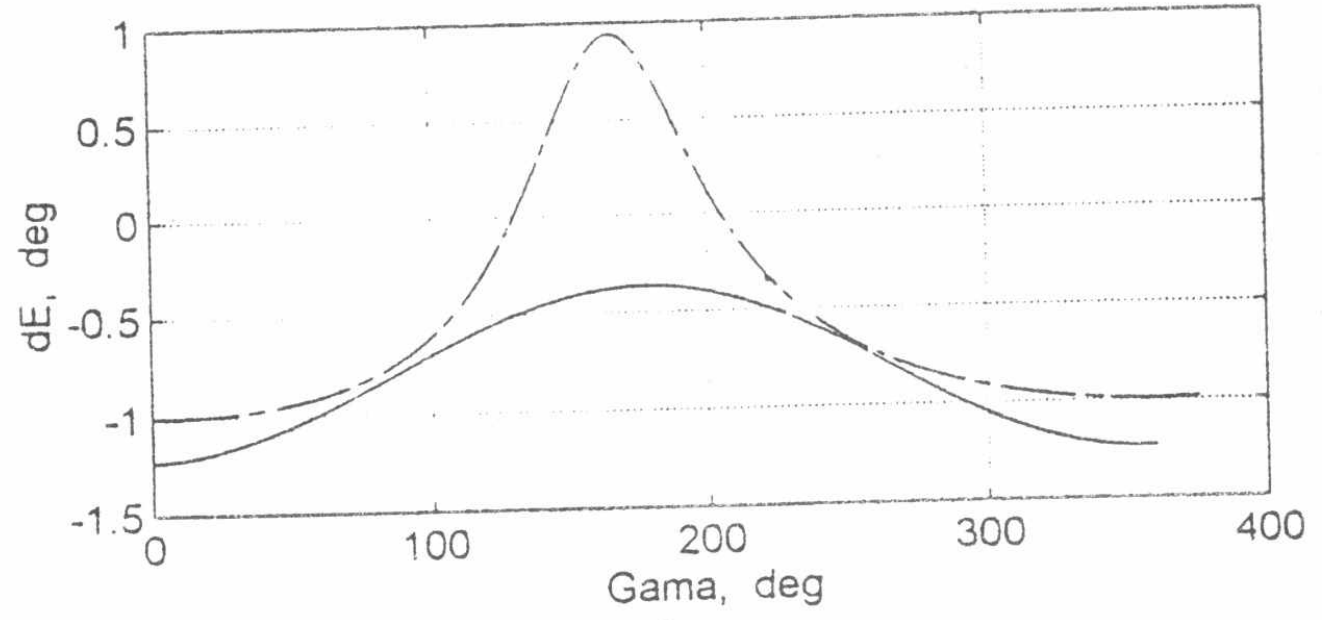

Fig. 14. Elevator deflection vs flight path angle.

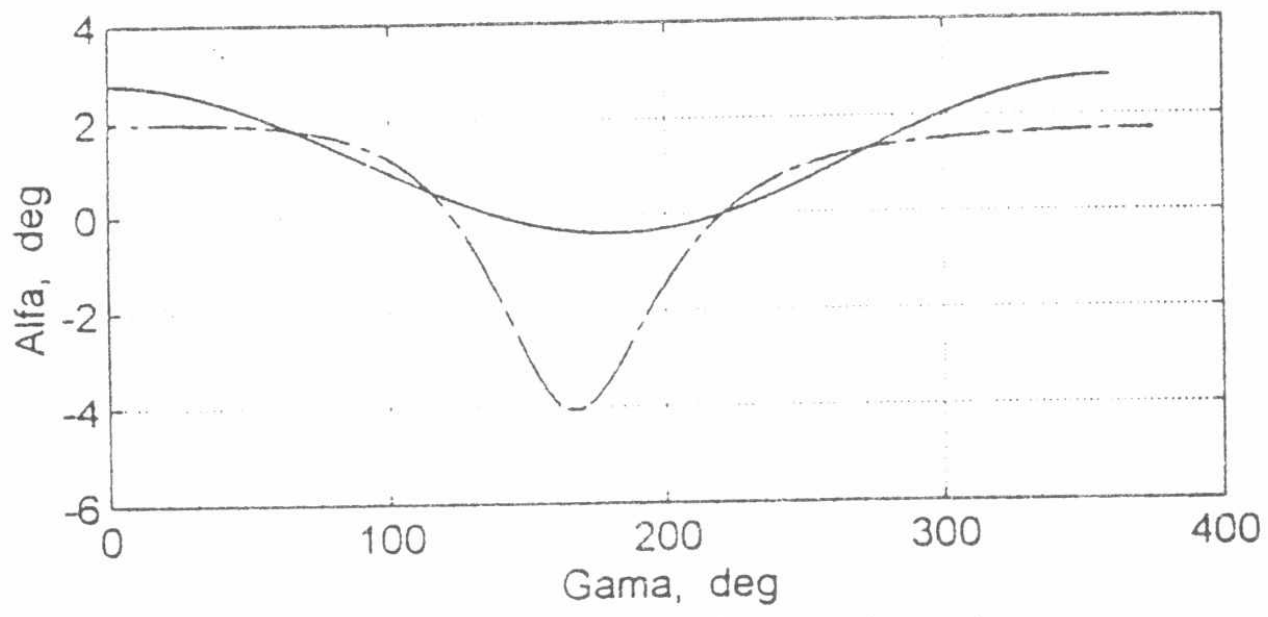

Fig. 15. Angle of attack vs flight path angle. 




Fig.16. Velocity vs flight path angle.

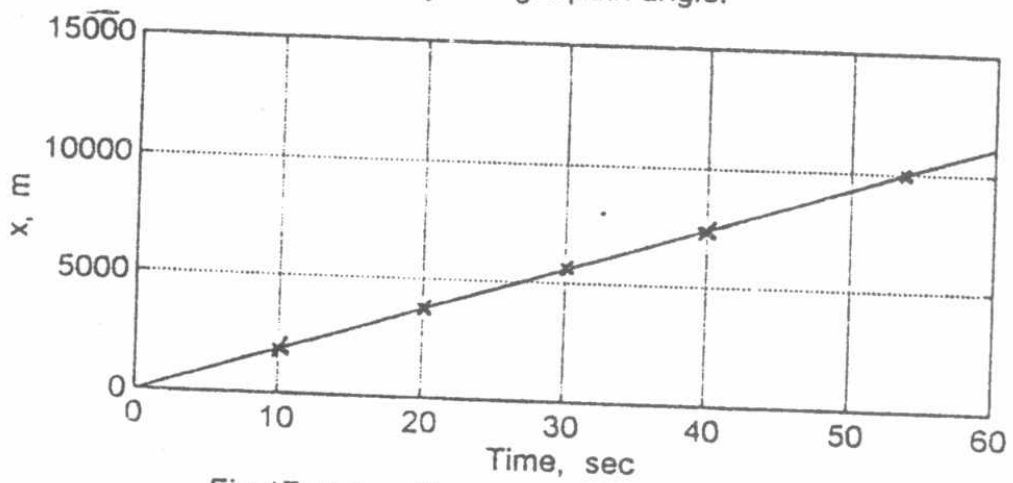

Fig.17. $x$-coordinate. time history.

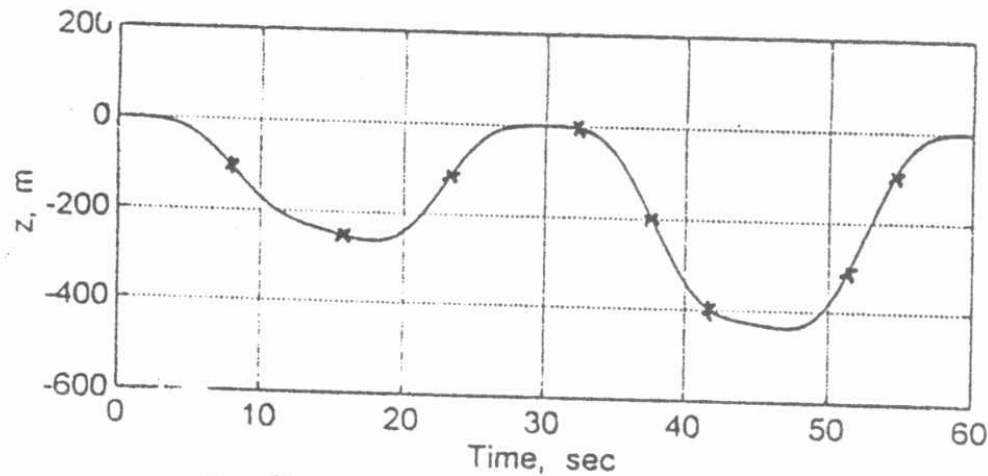

Fig. 18.z-coordinate. time history.

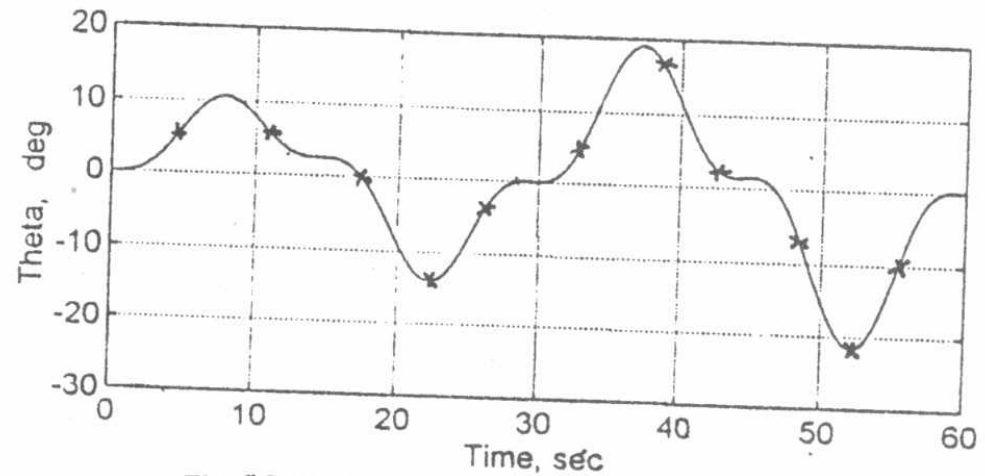

Fig. 19 Pitch angle time history 